\title{
Tendencies of Modern Physics.
}

THE Swiss Society of Natural Sciences met this year at Berne on August 24 to 27. The programme of the session comprised several discussions on questions of general interest, and papers of a more special character communicated to the various sections. The work was divided between the following sections: (I) Mathematics; (2) Physics; (3) Geophysics, Meteorology, and Astronomy; (4) Chemistry ; (5) Geology, Mineralogy, and Petrography; (6) Botany; (7) Zoology; (8) Entomology ; (9) Palæontology ; (Io) Anthropology and Ethnology; (I I) Medical and Biological Science; (I2) History of Medicine and Natural Science; (r3) Veterinary Science; (I4) Pharmacy ; (I5) Engineering History.

We cannot give here a detailed account of this annual event in Swiss science; we shall therefore confine ourselves to a résumé of the address of Prof. C. E. Guye, of Geneva, in opening the series of general discussions.

Taking the title, "The Tendencies of Modern Physics and the Conception of Matter," Prof. Guye first showed that modern physics was becoming more and more electromagnetic, discontinuous, and statistical. To these three characteristics, which have been sufficiently disconcerting to minds accustomed to the classical conceptions of the second half of the nineteenth century, there has now been added a fourth, of still more perplexing character, in the introduction of the principle of relativity. In adopting this principle physics has displayed a distinctly metaphysical tendency, which sometimes ventures to introduce into scientific discussion a dogmatic method of procedure. It is true that the difficulty is compensated by important advantages, resulting from the fact that the formulæ of relativity introduce more simplicity in the dynamics of very great velocities, and more unity between the various branches of physics.

After having shown how physics, like chemistry, has moved steadily along the path of discontinuity by the introduction of the atom of electricity and the theory of quanta, Prof. Guye spoke of the consequences of this discontinuity, which complicated greatly the explanation of phenomena apparently of the most simple character.

How, indeed, could one follow, by means of the equations of mechanics, the reciprocal actions of a nearly innumerable group of discontinuous elements (molecules, atoms, electrons)? This extreme complication which characterises the phenomenon, apparently so simple, when it is desired to study it intimately, led to the introduction of kinetic theories. The calculus of probabilities then came to the aid of physicists, powerless as they were to solve, by means of the equations of mechanics, the inextricable problems which were proposed to them. But the consequence of these kinetic theories is to lead us to conceive physico-chemical laws as statistical, so that we must picture physico-chemical determinism as a statistical determinism, to which the law of great numbers imparts all the appearance of infinite precision.

The progress of physics towards electromagnetism is particularly striking. The first decisive step along this path was made by Maxwell, to whom we owe the electromagnetic theory of light, which, universally accepted as it is to-day by physicists, unites in a systematic whole the phenomena of light and of electromagnetism. But this tendency to explain physical phenomena by the laws of electromagnetism has only served to make it still more accentuated. It has even attacked the mechanics which seemed to be the immutable basis of the old physics. To-day the fundamental postulate of mechanics-inertiacan be satisfactorily explained in terms of the properties of an electromagnetic field, and more and more intermolecular forces appear to be of electromagnetic nature (Debye, Keesom).

But the main reason for this constant evolution of physics towards electromagnetism is the work carried out particularly in England (Rutherford's school), which has exhibited it in a most convincing fashion. The material atom itself appears to be constituted entirely of charges of electricity, positive and negative (electrons), and all physical forces, with the exception of the mysterious force of gravitation, will thus be found, in the last resort, to be electric and magnetic forces.

In the second part of his address, Prof. Guye showed how the conception of matter, as defined by inertia, had evolved from Lavoisier to Einstein, and to the most recent work of Rutherford and Aston. Without committing ourselves positively to Prout's hypothesis, which would make the atomic weights of the elements integral multiples of that of a unique constituent-the atom of hydrogen-new developments point to a duality of ultimate material, the positive electron which is mainly responsible for the inertia of the atom, and the negative electron.

In short, the startling progress realised in physics during the last thirty years has reduced to naught all those fluid phantoms which we knew-imponderable electric and magnetic fluids; only the most tenacious among them-the aether-offers still a partial resistance.

Physicists have thus been led, little by little, to the idea of the materiality of electricity, and still more the formulæ of relativity point to the parallelism between inertia and energy; that is, to the fusion into a single principle of the two principles which govern all physical phenomena-the principle of the conservation of mass and that of the conservation of energy.

Such are the important results, not only from the scientific point of view, but also from that of our best philosophic culture, which modern research has brought forward during the course of the last thirty years.

\section{The Isothermal Frontier of Ancient Cities. ${ }^{1}$}

THE northern frontier of the Roman Empire is shown in atlases of ancient geography, and that of the Achæmenian Empire of the Persians and of the dynasties which succeeded in the Middle East. The.frontier of the ancient Chinese Empire has not been made similarly familiar, and in place of it there is the representation of the Empire of China as it

${ }^{1}$ Abstract of a paper by Dr. Vaughan Cornish read before Section E (Geography) of the British Association at Hull on Sept. I2. has been in mediæval and modern times. From this most of Manchuria, all Mongolia, and the Ili valley must be shorn off in order to get the Chinese northern frontier as it was under the Han dynasty in the beginning of the second century after Christ, the age of the Antonines in Europe. At this time, when the ancient civilisation of Eurasia was at the height of its culture and apparently at the maximum of its power, the northern frontier once controlled by 
the Persian Achæmenidæ was divided between the Parthians, capitalled at Ctesiphon, and the Kushan dynasty of the Yue-chi, capitalled at Peshawar. These four northern frontiers, Roman, Parthian, Kushan, and Chinese, were consecutive, forming an unbroken line from the mouth of the Rhine near the modern Katwyk in Holland, $52^{\circ} \mathrm{N}$., to the east coast of Korea in about $4 \mathrm{r}^{\circ} \mathrm{N}$. South of the line a vast array of established cities stretched for seven thousand miles across Eurasia, in some parts protected by natural barriers, in others defended by lines of masonry fortification. North of the line were the tents of nomads, huts of forest dwellers, and stockaded defences of earth and wood. In the northern part of modern Germany there were territories north of the line which the Romans had abandoned as untenable or unprofitable. South of the line in Eastern Europe was the district of Dacia which Augustus preferred not to touch, but Trajan was compelled to occupy. In this country the native people had in the interval begun to construct masonry fortifications.

In the course of an investigation of the geography of capital cities, it was found that this northern frontier of ancient cities, on the eve of the barbarian irruption, has, within narrow limits of variation, the same average temperature throughout. It is a true annual isotherm, not an isotherm reduced to sea level. Along the European part is a line of modern cities with meteorological observatories. The annual temperatures of eight of these, strung out along the length, has an average of $48^{\circ} \cdot 6 \mathrm{~F}$. Asia is not well off for meteorological records near the line on the south, and the second table consists of a list of towns mostly under Russian rule just north of the line where proper records have been kept. It will be observed, therefore, that their temperatures are rather lower than that along the frontier of the ancient cities. The average temperature of these eight towns north of the line is $47^{\circ} \cdot 4 \mathrm{~F}$. A very long gap in these towns occurs between Kuldja and Mukden, but the record for the Lukchun depression in Chinese Turkestan, a little south of the frontier yields a not inconsistent figure, if corrected for the general height of the surrounding country, and that of Peking is not discordant. Further east the generalised isotherm of $48^{\circ} \cdot 5 \mathrm{~F}$. reaches the eastern coast of Korea in about $4 \mathrm{I}^{\circ} \mathrm{N}$. (somewhat north of

the peninsula portion of the country) which cannot be very far from the frontier of its ancient cities.

In the detached Roman possession of Britain the inner and principal line of fortification had its western terminal at Carlisle, where the temperature is $47^{\circ} \cdot 8 \mathrm{~F}$. Eastwards of the continent of Eurasia the conquest of the Japanese islands by their present masters was only completed at a much later date than that under consideration, but the Japanese derived their culture from ancient China (mainly through Korea) and it may therefore be significant that they were content to conquer, without colonising, Yezo, the northern island, and that what is reckoned by the Japanese as Japan proper, and is called by them "Old Japan" does not include Yezo but stops short with Honshiu, the mainland, and that the annual isotherm of $48^{\circ} .5 \mathrm{~F}$. traverses the strait of Tsugaru which separates Old Japan from Yezo.

The fact that the annual temperature along this immense line only varies within remarkably narrow limits cannot be reasonably contested. If it be the case that desiccation has occurred generally in Asia along this line since the second century of our era its probable effect would be to lessen the winter and raise the summer temperature, leaving the annual temperature much the same.

The coincidence of frontier and true isotherm is not a mere consequence of east and west barriers of mountains, inland seas, and rivers, for these had to be supplemented by long lines of fortification. Neither was it due to unsuitability of the southern country to pastoral peoples, for in Asia there was much coveted grazing land south of the settled frontier. Precisely how far this coincidence is significant it is yet difficult to say.

\begin{tabular}{|c|c|c|c|c|c|c|}
\hline \multicolumn{3}{|c|}{$\begin{array}{l}\text { Annual Temperatures along } \\
\text { the Frontier in Europe. }\end{array}$} & \multicolumn{4}{|c|}{$\begin{array}{c}\text { Annual Temperatures north of } \\
\text { the Frontier in Asia. }\end{array}$} \\
\hline & & ${ }^{\circ} \mathrm{F}$ & & & & ${ }^{\circ} \mathrm{F}$. \\
\hline Carlisle & • & $47^{\circ} 8$ & Stavropol & • & • & 47 \\
\hline Utrecht & - & $47 \cdot 8$ & Astrachan & $\cdot$ & & 50 \\
\hline Cologne & $\bullet^{\circ}$ & $50 \cdot 2$ & Kazalinsk & . & 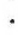 & $4^{6 \cdot 5}$ \\
\hline Ratisbon & . & $46 \cdot 6$ & Aulieata & . & • & $5 x \cdot 5$ \\
\hline Vienna & • & $4^{8 \cdot 8}$ & Narynsk & • & • & 44 \\
\hline Buda-Pesth & . & $49 \cdot 8$ & Vyerni & $\cdot$ & * & $46 \cdot 5$ \\
\hline Debreczin & . & $49 \cdot 3$ & Kuldja & & & $48 \cdot 5$ \\
\hline Odessa & $\cdot$ & $4^{8} \cdot 5$ & Mukden & $\bullet$ & 列 & $45^{\circ}$ \\
\hline Mean & ${ }^{\circ}$ & $4^{8 \cdot 6}$ & Mean & & & $47^{\circ}$ \\
\hline
\end{tabular}

\section{The Mechanism of the Cochlea.}

M OST medical students have probably felt that current physiological teaching provided them with only a hazy conception of the mechanism for hearing in the cochlea. Helmholtz put forward the view that this organ contained a series of resonators, which were differentiated like a set of piano strings, so that each string vibrated only in response to one particular note. It will be remembered that the cochlea forms a spiral, which when unwound consists of two chambers, placed one above the other, and separated by the basilar membrane. At one end (the base) of the cochlea, in the wall of the upper chamber, is the window which is set in vibration by the middle ear, while in the wall of the lower chamber is a similar window whose function is to prevent the pressure from changing inside the cochlea when the upper window moves. Both chambers contain fluid, and, at the other end (the apex) of the cochlea, the chambers unite, for the basilar membrane ceases just short of the apex.

The suggestion that the fibres of the basilar membrane can act as a resonating system has been current since it was pointed out that their length (measured across the canal) varied continuously from the base to the apex. Now the fibres of a resonating system must obey the laws which govern vibrating strings, so that $n$, the number of vibrations of a string per sec., $=\frac{\mathbf{I}}{2 l} \sqrt{\frac{t}{m}}$, where $l$ is the length of a fibre, $t$ is the tension, and $m$ is the mass per unit length. Gray showed in I9oo that the tension of the fibres of the basilar membrane also varied from the base to the apex, for while the spiral ligament which attached the membrane to the outer wall of the cochlea was very dense near the base, it was, on the contrary, very slender near the apex. We know, therefore, that the fibres of the basilar membrane are differentiated for tension and length, so that the short fibres near the base are under high tension, and the long ones near the apex are under low tension. To complete the requirements of the formula for vibrating strings, it is only necessary to discover a system by which the fibres are differentiated for mass, which differentiation must, as the formula

$$
\text { NO. } 2764 \text {, VOL. IIO] }
$$

